\title{
Circulating microRNA-194 and microRNA-1228 Could Predict Colon Cancer Proliferation via Phospho S6 Modulation
}

\author{
Sergiu Pasca ${ }^{1}$, Calin Ionescu ${ }^{1,2}$, David Andras ${ }^{1}$, Dan Eniu ${ }^{1,3}$, Mihai-Andrei Mureșan $^{1,3}$, Lorand Magdo ${ }^{1}$, Ancuta Jurj $^{1,4}$, \\ Lajos Raduly ${ }^{1,4}$, Roxana Cojocneanu ${ }^{1,4}$, Bobe Petrushev ${ }^{5,6}$, Florin Zaharie ${ }^{1,5}$, Alexandru Irimie ${ }^{1,3}$, Ioana Berindan-Neagoe ${ }^{1,4,7,8}$, \\ Mihai-Stefan Muresan ${ }^{1,2}$
}

1) Iuliu Hatieganu University

of Medicine and Pharmacy,

Cluj-Napoca;

2) $5^{\text {th }}$ Surgical Department,

Municipal Hospital, Cluj-

Napoca;

3) Department of Surgery, Ion

Chiricuta, Oncology Institute,

Cluj-Napoca;

4) Research Center for

Functional Genomics,

Biomedicine and Translational

Medicine, Cluj-Napoca;

5) Octavian Fodor Regional

Institute of Gastroenterology

and Hepatology, Cluj-Napoca;

6) Department of Pathology,

Regina Maria Laboratory,

Cluj-Napoca;

7) MEDFUTURE-Research

Center for Advanced

Medicine, Cluj-Napoca;

8) Department of Functional

Genomics and Experimental

Pathology, Ion Chiricuta

Oncology Institute, Cluj-

Napoca,

Romania

\author{
Address for correspondence: \\ Calin Ionescu \\ $5^{\text {th }}$ Surgical Department, \\ Municipal Hospital, Cluj- \\ Napoca; Department of \\ Surgery, Iuliu Hatieganu \\ University of Medicine and \\ Pharmacy, Cluj-Napoca, \\ Romania \\ ionescu_calincj2002@yahoo.com
}

Received: 25.11 .2019

Accepted: 10.06 .2020

\section{ABSTRACT}

Background \& Aims: Although colon cancer has a decreasing incidence trend in Europe, because of its still high frequency and not fully understood pathogenesis, this malignancy still remains a subject of intense research. The aim of this study was to investigate the role of microRNA-194 and microRNA-1228 in colon cancer proliferation.

Methods: RNA was extracted from patients with colon cancer with or without advanced disease and microRNA expression levels were determined through qRT-PCR. Assays were performed on HCT116 cell line and included qRT-PCR, western blotting and cell counting.

Results: We observed that both microRNAs 194 and 1228 were altered in patients with colon cancer compared with healthy individuals. We observed a lower expression of both microRNA-194 and microRNA-1228 in patients with advanced colon cancer. To validate their pathogenetic role we performed viability and invasion assays on HCT116 cell line transfected with mimics or inhibitors of the mentioned microRNAs, with observable changes in viability and invasion. Furthermore, to determine the altered signaling induced by these microRNAs, we performed western blotting for phospho S6 on HCT116 cells transfected with mimic and inhibitor of the above-mentioned microRNAs with observable differences.

Conclusion: In the current study we have shown that both microRNA-194 and microRNA-1228 alteration was correlated with the presence of advanced colon cancer, a fact that was further validated in vitro through an invasion assay. Moreover, we have also shown that their effect might be mediated through phospho S6 expression.

Key words: microRNA-194 - microRNA-1228 - colon cancer - biomarkers.

Abbreviations: CC: colon cancer; PI3K: phosphatidylinositol 3-kinase; PTPRN2: protein tyrosine phosphatase receptor type N2; qRT-PCR: quantitative reverse-transcriptase polymerase chain reaction.

\section{INTRODUCTION}

In Europe, colon cancer (CC), represented by its most prevalent histology, adenocarcinoma, has a high incidence in both men and women, being the third most frequent cancer in men and second in women $[1,2]$. Considering mortality, $\mathrm{CC}$ is second only to lung cancer in men and third in women, after breast and lung cancer. For more than 20 years, CC has presented a constant decline in incidence and mortality and a certain convergence on incidence and mortality rates between different countries $[1,2,4]$. This is probably due to the homogenization of medical culture and to a better access to diagnosis and therapeutic tools [1-3]. Moreover, the improved survival rates could also be indirectly linked to the continuous advancements in intensive care medicine $[4,5]$.

More than ten years have passed since there has been proof that microRNAs have an impact in cancer biology; nowadays these molecules present potential as biomarkers or as therapeutic targets [6]. These are 18-25 nucleotide-long RNAs with a regulatory role generally thought as acting at the level of 3'UTR (untranslated region) of mRNAs, but also having other mechanisms of action, e.g. binding to promoter sites [9-11].

Two examples of microRNAs' effects on tumor biology are represented by cell proliferation [12-14] and metastasis $[15,16]$. Although there is already published works on these matters, there is still need for their exploration and validation through studies in different centers. Since 2006, Calin et al. 
[6], hypothesized that between CC and rectal cancer, different microRNA signatures can be identified. Out of the specific species of microRNA identified and described in their work, some could behave as tumor promoters (oncogenes), whilst others could have the reverse outcome, being tumor inhibitors/ suppressors. Significant differences and outcomes have been found for some species when describing CC whereas when describing rectal cancer, the same microRNA species were associated with striking differences in altered pathways. Similar conclusions were reported in subsequent studies [16, $17,21]$.

The aim of the present study was to investigate the role of microRNA-194 and microRNA-1228 in CC proliferation.

\section{METHODS}

\section{Subjects}

In the present study we have included plasma samples from 55 patients with CC (primary adenocarcinomas situated anywhere between the ileo-cecal valve and the superior rectum, including the recto-sigmoid junction, irrespective of clinical or imaging staging) out of the 101 colorectal patients addmited between January 2014 and January 2015 at the Surgical and Gynaecologycal Oncology Department, Ion Chiricuta Oncology Institute, Cluj-Napoca (IOCN), Romania. From the selected 55 patients, thirteen presented with either advanced loco-regional disease or distant metastases to the liver at initial diagnosis (ten patients with advanced loco-regional disease, and three patients with distant liver metastases). Sixteen other plasma samples collected from healthy volunteers were used as the control set. The patients were enrolled in the study after signing an informed consent, and their data was protected in accordance with EU confidentiality regulations. The study was conducted according to the declaration of Helsinki and was approved by the Ethics Committee from IOCN (60/26.01.2017). The results shown here are in part based upon data generated by the TCGA Research Network: https://www.cancer.gov/tcga. All subsequent assays, arrays and in vitro studies were performed at the same time for the selected plasma specimens. The findings of this study were not analyzed in correlation to pathology findings, tumor burden, prognostic factors, clinical outcomes or overall survival of the selected patients. The only relationship analyzed with the identified microRNA species was the presence or absence of advanced colon cancer at diagnosis.

\section{MicroRNA selection}

A microarray analysis using the one-color protocol from Agilent ${ }^{\oplus}$ for miRNA expression was used in order to determine the most up/down regulated microRNAs. The obtained data were assessed with GeneSpring ${ }^{\circledast}$. Furtheron, we have used the IPA ${ }^{\star}$ (Ingenuity Pathway Analysis) software to identify the signaling pathways influenced by the most relevant microRNAs, as briefly described by Raduly et al. [17]. After determining the microRNAs species that were up/downregulated, we performed a PubMed and Cochrane database search of literature articles with references/results concerning the identified microRNAs species.

\section{Cell culture}

The CC cell line, HCT-116 Dicer was used in this study. The cells were grown in an atmosphere with the following characteristics: humidified atmosphere, $37^{\circ} \mathrm{C}$ air, $5 \% \mathrm{CO} 2$. The culture media used was McCoy's (Gibco) supplemented with $10 \%$ fetal bovine serum (FBS) (Gibco), $100 \mathrm{UI} / \mathrm{ml}$ penicillin and $100 \mathrm{mg} / \mathrm{ml}$ streptomycin (Gibco).

\section{RNA extraction}

Total RNA extraction was performed using TRIzol reagent (Invitrogen), as previously described by Peng $\mathrm{H}$ et al. [11].

\section{Transfection}

HCT 116 cells $\left(2 \times 10^{\wedge} 5\right.$ cells/well $)$ were transfected with microRNA-194 mimic or inhibitor or with microRNAR-1228 mimic or inhibitor using Lipofectamine as previously described [14]. RNA was harvested at 24, 48 and 72 hours. Proteins were harvested at 24 hours.

\section{Cell counting}

The treated cells were counted at 24, 48 and 72 hours using a hemocytometer and the Countess Automated Cell Counter (Invitrogen).

\section{MTT assay}

The MTT assay was carried out as previously described by Petrushev et al. [18].

\section{Microscopy}

The cell images were taken using a Leica S80 inverted microscope.

\section{Quantitative reverse-transcriptase polymerase chain} reaction (qRT-PCR)

MicroRNA expression was quantified in patients' samples and in cell culture using qRT-PCR. The assay was carried out as previously described [19]. The reactions were carried out in technical duplicates.

\section{Western blotting}

Western blotting was performed as previously described, with the exception of the primary antibody, which was either anti-phospho S6 or anti-actin antibody [19].

\section{Statistical analyses}

The statistical analysis was performed using GraphPad Prism 5.0 (GraphPad Software INC., CA, USA) and R 3.5.3. cBioPortal was used to download the relevant data. The Shapiro-Wilk test was used to assess the normality of distribution. The t-test was used to assess differences between two normally distributed variables. The Wilcox test was used to assess the differences between two non-normally distributed variables. One-way ANOVA was used to assess differences between multiple groups. If one-way ANOVA was statistically significant, the exact differences were assessed using Turkey's multiple comparison test. Correlation between two nonnormally distributed variables was assessed using the Spearman test. A p value below 0.05 was considered significant. 


\section{RESULTS}

\section{Patients' characteristics}

The mean age, irrespective of gender, for the 55 selected patients, was 61 years. Thiry-one were males (56\%) and 24 females (44\%); 25 patients had CC (ascending, transverse or descending colon) and 30 patients had recto-sigmoid junction cancer.

\section{MicroRNA selection}

The analysis performed to compare the CC plasma samples versus healthy controls generated a total number of 335 microRNAs, which showed an altered expression with statistical significance ( $\mathrm{p}$ value $<0.05$, fold change $>2.0$ ). From these microRNA species, 61 were upregulated and 273 were downregulated (Fig. 1) [17]. When further analyzed, as expected, the most frequently altered biological processes were associated with cancer cell invasion, cell growth and migration, apoptosis and inflammation. From these 335 microRNAs, showing altered expression with statistical significance, we have selected microRNA-1228 as the upregulated microRNA and microRNA-194 as the downregulated one for further analyses. The two microRNAs we chose to study were selected based on the previous microarray data [17] and from an extensive literature review (approximately 850 articles identified on PubMed and Cochrane Library with data regarding the 335 microRNAs species with altered expression), which showed scarce data for these two microRNAs. As expected, the selected microRNA species' influence was mostly prevalent when investigating the most common altered pathways in cancer represented by those involved in inflammation, migration and cell growth.

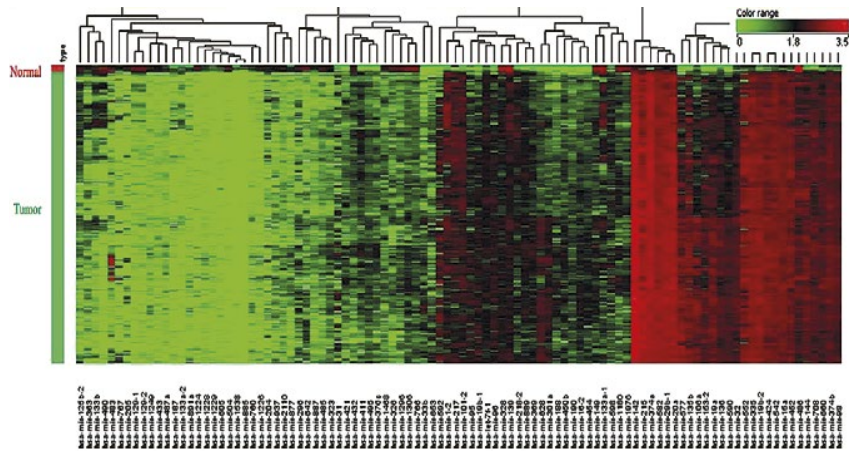

Fig. 1. Heat map of TCGA data for microRNAs in our selected CC patients.

\section{MicroRNA expression in CC patients}

qRT-PCR on patients' microRNA samples showed statistically significant differences in microRNA-194 and microRNA-1228 expression between patients with locally advanced/metastatic disease versus patients without advanced disease. Samples from patients with locally advanced disease or liver metastasis revealed lower expression levels for both microRNAs (Fig. 2).

\section{Transfection effect on cell proliferation}

The effect of microRNA-194 mimic and microRNA-1228 inhibitor on cell proliferation was assessed at 24, 48 and 72

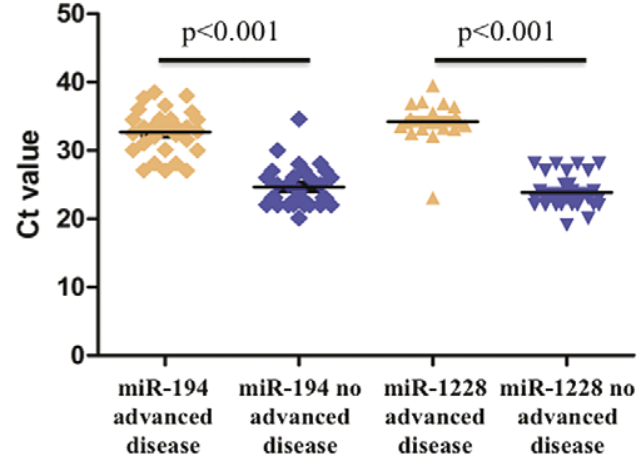

Fig. 2. Differences in microRNA-194 and microRNA-1228 expressions between colon cancer patients with and without advanced disease.

hours through cell counting and MTT assay (Fig. 3 A-F). Using the MTT assay, we observed that at all time points microRNA-1228 inhibition determined a statistically significant higher cancer cell viability, whereas microRNA-194 upregulation (via its mimic) determined a lower viability at all time points compared to control (Fig. 3 A-C).

Unfortunately, when cell counting was assessed, the results we have obtained were congruent with those achieved at cell viability assessment (see above) only at 24 hours, whereas 48 and 72-hours analyses did not show similar trends. To detail, microRNA-194 mimic transfected cells had a statistically significant lower proliferation rate $(\mathrm{p}=0.0316)$ when compared to control cell lines. Another statistically significant effect on cell proliferation was observed at 72 hours; microRNA-1228 inhibitor significantly inhibited HCT116 cell line proliferation compared to the control ( $\mathrm{p}=0.0161)$ (Fig. $3 \mathrm{~F}$ ), but without striking differences when compared to microRNA-194 mimic transfected cells.

\section{Cell invasion potential}

In accordance with the cell proliferation assay, cells transfected with microRNA-1228 mimic have an increased proliferation rate and invasion potential, as shown in Figs. $4 \mathrm{~A}$ and $4 \mathrm{~B}$. On the other hand poor cell proliferation and invasivity for HCT-116 cells transfected with microRNA-194 mimic is shown. As underlined in the proliferation data, this qualitative assay proved obvious higher proliferation and invasivity of microRNA-1228 transfected cells with lower levels of cells in such status, when using microRNA-194 mimic for transfection. As proven in the proliferation data, the differences between cells transfected with non-specific mimic and those transfected with an inhibitor lack statistical significance. We can thus conclude that, for the time being in our case series, microRNA-194 might potentially be an inhibitor of growth and invasion of CC cells, whereas microRNA-1228 could potentially promote proliferation and invasivity of CC cells.

\section{Effect of microRNA on protein expression}

Using Western blotting, we identified a significant difference in phospho 66 expression. In microRNA-194 mimictransfected cells, phospho S6 had lower expression; the same relevant, more poignant observation can be made when using microRNA-1228 inhibitor for HCT-116 cell line transfection. On the other hand, when transfection was performed using 
A

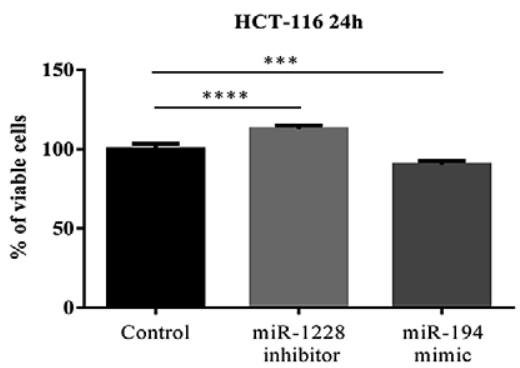

C

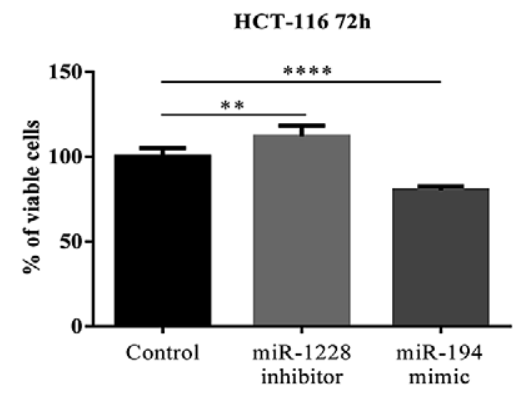

E

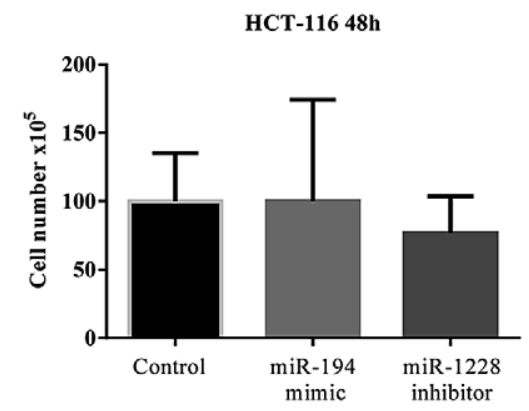

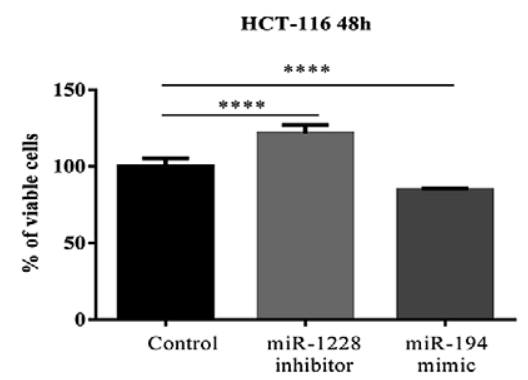

$\mathrm{D}$

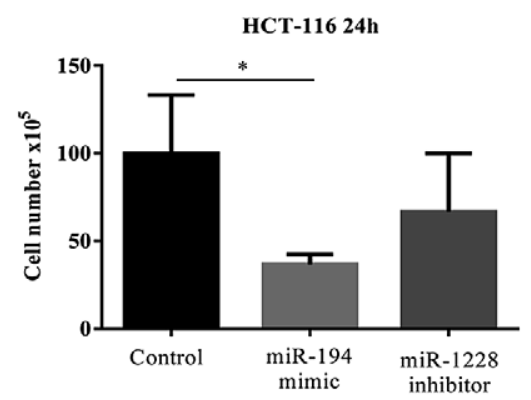

F

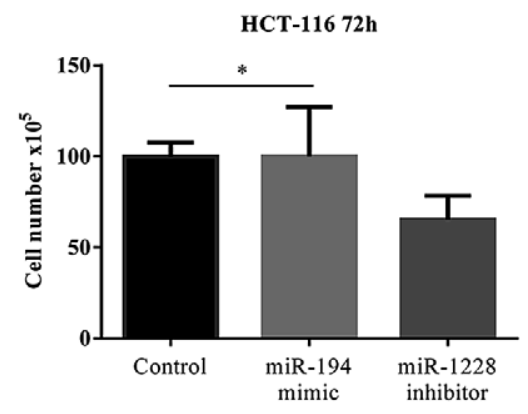

Fig. 3. A-F. microRNA influence on cell proliferation objectified with cell viability (Figure $3 \mathrm{~A}-\mathrm{C}$ ) and cell number assessment (Figure $3 \mathrm{D}-\mathrm{F}$ ). $\mathrm{p}^{*}<0.05 ; \mathrm{p}^{* *}<0.01 ; \mathrm{p}^{* *}<0.001$; $\mathrm{p}^{* * * *}<0.0001$.

microRNA-1228 mimics or microRNA-194 inhibitors, phospho 66 was upregulated (Fig. 5).

\section{DISCUSSION}

In the current study we have shown that both microRNA-194 and microRNA-1228 have a role in CC cell proliferation and metastasis, having the potential to act as biomarkers.

MicroRNA-194 has been studied before in CC and the general trend shows that an overexpression of the present
microRNA has inhibitory effects on cell proliferation and metastasis. These reported results are similar to the ones presented in our current study. The major role played by microRNA-194 in CC has been described before in an important article by Jung et al. [20]. Wang et al. [21] showed that microRNA-194 could be used as a predictor for adenoma recurrence after polypectomy in patients with advanced colorectal adenoma. In their study, lower microRNA-194 levels were associated with a higher
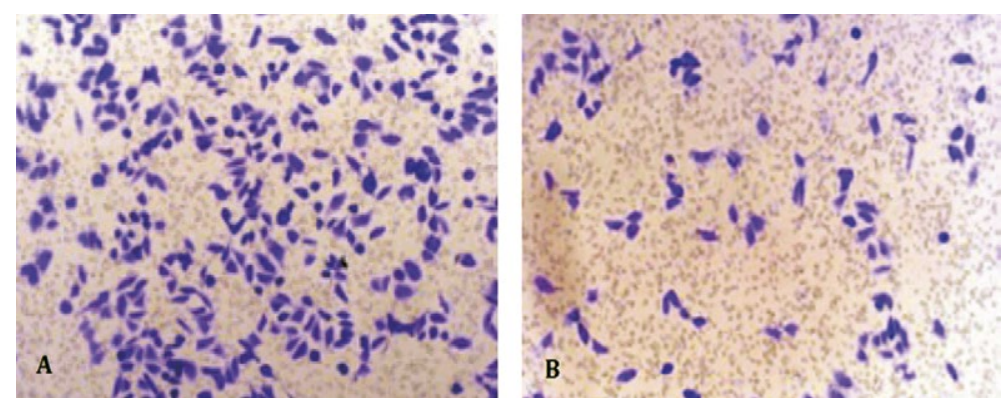

Fig. 4. In vitro Matrigel cell proliferation assay: A) bloat microRNA-1228mimic HCT-116 transfected cells; B) bloat microRNA-194 mimic HCT-116 transfected cells. 


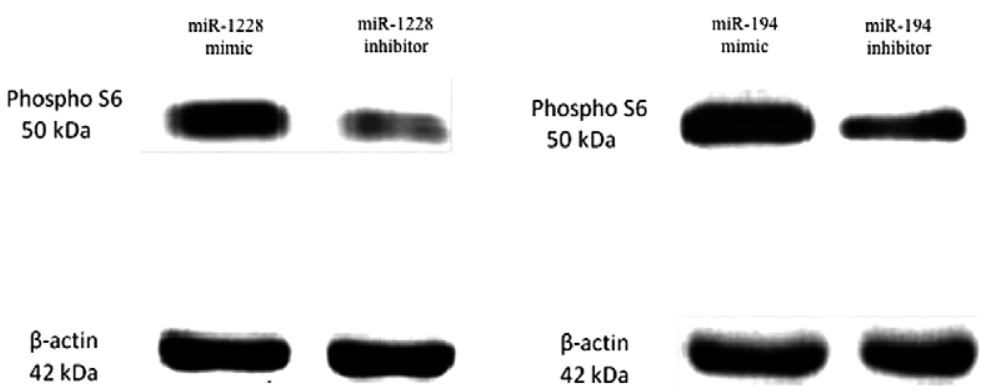

Fig. 5. Western blotting for phospho S6 expression in HCT-116 cell line.

recurrence rate, emphasizing the role of the microRNA-194 in CC's proliferation potential.

Chang et al. [22] showed the negative correlation between microRNA-194 and high-mobility group AT-hook 2 (HMGA2). Their study reported the bidirectional influence between these two molecules and the inhibitory role of re-expression of microRNA-194 in cell survival rates and epithelial to mesenchymal transition. These results reconfirm microRNA's-194 importance in tumor proliferation and metastasis [22].

Wang et al. [23] provided evidence that in CC patients microRNA-194 is commonly repressed and furthermore, that microRNA-194 downregulation in their cohort of CC patients was inversely associated with tumor size and TNM stage. They also provided functional evidence that overexpression of microRNA-194 in CC cell lines inhibited growth both in vitro and in a mouse subcutaneous xenograft model [23]. Still, Zhao et al. [24] showed in an in vitro and in vivo model that overexpression of microRNA-194 inhibited cell invasion, viability and suppressed xenograft growth, whereas Sundaram et al. [25] showed a contrary effect of microRNA-194 to what was presented until now in the present paper. Their study revealed that microRNA-194 overexpression has a thrombospondin-1 mediated effect on an in vitro and in vivo level in boosting angiogenesis (microvascular densities and vessel sizes) and facilitating tissue repair. The opposite expression and effects are also reported in their study showing another influence of this microRNA [25].

Correlating our results cencerning microRNA-194, with the above-mentioned studies, we can conclude that this microRNA plays a significant role in decreasing tumor cellularity, acting as a tumor suppressor microRNA, when upregulated. Future studies need to address this microRNA in order to adequately establish its apoptosis promotion capabilities in different types of cancer histology. Bearing in mind the promising results regarding microRNA-194, and comparing these results with the available literature, we can further firmly hypothize that this microRNA's downregulation can be regarded as a marker of cell proliferation and metastasis with potential in predicting CC evolution.

To the best of our knowledge, at the time when this paper was written, there were no validation studies on microRNA-1228 and CC, even if promising results were reported concerning its dysregulated expressions in other carcinomas.

Zhang et al. [26] hypoththetized that microRNA-1228 upregulation in an in vitro and an in vivo context promotes cell proliferation and metastasis in human hepatocarcinoma.
These results are similar in the aspect of cell proliferation with our results at 72 hours, showing that an inhibition of microRNA-1228 inhibits cell growth, but on the other hand, they are in disagreement with our results showing that microRNA-1228 downregulation is associated with liver metastasis. We have to bear in mind the fact that these are different types of carcinomas and that the metastasis potential is assessed differently in the two studies. Some additional mechanisms might coexist when taking into account a patient cohort discriminating the primary tumor's histology [26]. One other aspect, to take into account in our study, is the fact that inconsistent results were obtained for our transfected cell line, when comparing transfected cells' viability to transfected cells' cell count. This could be related to either physical loss of the transfected cells on the Countess Automated Cell Counter (Invitrogen) or to misinterpretation of cells considered to be viable at counting, being in fact in a pre-apoptotic state.

Lin et al. [27] shed some light in the context of microRNA's-1228 expression in breast cancer context. They reported a higher proliferation and metastasis potential in a patient cohort as well as in an in vitro model. This study's results [27] are concurrant with data reported by Zhang et al. [26] and to our own data, agreeing to the proliferative effect of microRNA's-1228 upregulation has.

Correlating our results concerning microRNA-1228, to the above-mentioned studies, we can conclude that this microRNA plays a significant role in increasing tumor cellularity and potential invasiveness, acting as an oncogene microRNA, when overexpressed/upregulated.

Phospho S6 is a ribosomal protein that has been previously described as being a potential target to be inhibited for desensitization of tumor cells to chemotherapy, being presented as upregulated in tumor tissues and having a role in proliferation [28-32]. This was also described as beind responsive to phosphatidylinositol 3-kinase's (PI3K) activation in CC [33]. Moreover, in MirTarBase, it was obseved that microRNA-1228 targets protein tyrosine phosphatase receptor type N2 (PTPRN2), while microRNA-194 targets protein tyrosine phosphatase non-receptor types 12 and 13 , which are known to influence the activation of PI3K/Protein kinase B (PKB) (PI3K/AKT) pathway [34-36]. The previous statements stand as arguments for our choice of phospho $\mathrm{S} 6$ in the Western Blot analysis.

To our knowledge, this is the first study showing that microRNA-1228 inhibition downregulates phospho S6 expression, both compared to microRNA-1228 mimic and microRNA-194 mimic. 
One potential drawback of our study was not determining baseline values for phospho S6 levels in negative controls. In this matter, we have to admit the fact that no direct causality between the described microRNA species and their impact over phospho $\mathrm{S} 6$ has been identified. Future trials and quantification of results are required to truly describe the relationship between the microRNA species mentioned in our study and the impact on $r$ the expression of the ribosomal protein, phopsho S6.

To sum up, even though microRNA-1228 is suspected as having a positive influence over cell proliferation when taking into consideration other carcinomas, our own data scarcely confirm this hypothesis, so, for the time being, we are not in favor of including this microRNA in a clinical context.

Nonetheless, it has to be mentioned that the implication of microRNAs in CC has been extensively studied, with others describing the importance of a 5-microRNA panel in assessing the metastasis of CC to lymph nodes [37]. Others have attempted to show an association between polymorphisms occuring in microRNAs and the development of CC in the European population but have not observed any correlation [38]. Additionally, when discussing a change in microRNA expression, epigenetic changes should also be taken into consideration as it has been shown that microRNA-137 epigenetic silencing has a role in gastric cancer [39].

Finally, yet importantly, we have to be honest with our reported results, concerning the cell invasion potential of the investigated microRNAs. One important limitation of the current study concerns the invasion potential of the selected microRNAs. Even if the cell proliferation assays were backedup by negative controls showing promising results, cell invasion potential assays of the HCT-116 microRNA transfected cells were not compared to other tumour cell lines, and the only available control was assessed through non-specific mimic transfection. This issue has to be addressed through further studies comparing our initial invasion assays to additional ones, performed on at least one additional cell line, used as a negative control. Furthermore, when we investigated phospho S6 expression in the microRNA-transfected cells, using Western blotting, we did not use a negative control, so results must be interpreted accordingly.

One other minor limitation of our reported results is the limited number of patients included in the study, leading to a limited and unequivalent number of samples subjected to analyses. For this reason, yet again, we wholeheartedly emphasize the need of further larger similar studies.

\section{CONCLUSIONS}

Both microRNA-194 and microRNA-1228's alterations could correlate with the presence of advanced CC. This statement is backed-up by most of the in vitro invasion assays. Phospho S6 expression could be the responsible protein involved in their altered pathway.

Conflicts of interest: None to declare.

Authors' contribution: S.P., C.I., M.S.M. wrote and corrected the paper. D.A., D.E., C.I., A.I, M.A.M., M.S.M and F.Z. were responsible for patients' selection, specimen harvesting and data gathering. S.P.,
L.M., A.J., R.C, R.L., B.P and I.B.N. were responsible for the in vitro experiments (cell cultures, PCR, WB, mollecular biology arrays). M.S.M., C.I., A.I, F.Z. and I.B.N. were been responsible to the general supervision and provided funding for the experiments.

\section{REFERENCES}

1. Malvezzi M, Carioli G, Bertuccio P, et al. European cancer mortality predictions for the year 2018 with focus on colorectal cancer. Ann Oncol 2018;29:1016-1022. doi:10.1093/annonc/mdy033

2. Siegel RL, Miller KD, Jemal A. Cancer statistics, 2016. CA Cancer J Clin 2016;66:7-30. doi:10.3322/caac.21332

3. Ferlay J, Colombet M, Soerjomataram I, et al. Cancer incidence and mortality patterns in Europe: Estimates for 40 countries and 25 major cancers in 2018. Eur J Cancer 2018;103:356- 387. doi:10.1016/j. ejca.2018.07.005

4. Abbott TEF, Ahmad T, Phull MK, et al. The surgical safety checklist and patient outcomes after surgery: a prospective observational cohort study, systematic review and meta-analysis. Br J Anaesth 2018;120:146-155. doi:10.1016/j.bja.2017.08.002

5. International Surgical Outcomes Study (ISOS) group. Prospective observational cohort study on grading the severity of postoperative complications in global surgery research. Br J Surg 2019;106:e73-e80. doi:10.1002/bjs.11025

6. Calin GA, Croce CM. MicroRNA signatures in human cancers. Nat Rev Cancer 2006;6:857-866. doi:10.1038/nrc1997

7. Chekulaeva M, Filipowicz W. Mechanisms of miRNA-mediated post-transcriptional regulation in animal cells. Curr Opin Cell Biol 2009;21:452-460. doi:10.1016/j.ceb.2009.04.009

8. Place RF, Li LC, Pookot D, Noonan EJ, Dahiya R. MicroRNA-373 induces expression of genes with complementary promoter sequences. Proc Natl Acad Sci U S A 2008;105:1608-1613. doi:10.1073/pnas.0707594105

9. Jurj A, Pop L, Petrushev B, et al. Exosome-carried microRNA-based signature as a cellular trigger for the evolution of chronic lymphocytic leukemia into Richter syndrome. Crit Rev Clin Lab Sci 2018;55:501-515. doi:10.1080/10408363.2018.1499707

10. Muresan M, Zaharie F, Bojan A, et al. MicroRNAs in liver malignancies. Basic science applied in surgery. J BUON 2015;20:361375.

11. Peng H, Ishida M, Li L, et al. Pseudogene INTS6P1 regulates its cognate gene INTS6 through competitive binding of miR-17-5p in hepatocellular carcinoma. Oncotarget 2015;6:5666-5677. doi:10.18632/ oncotarget. 3290

12. Calin GA, Croce CM. MicroRNA signatures in human cancers. Nat Rev Cancer 2006;6:857-866. doi:10.1038/nrc1997

13. Schetter AJ, Leung SY, Sohn JJ, et al. MicroRNA expression profiles associated with prognosis and therapeutic outcome in colon adenocarcinoma. JAMA 2008;299:425-436. doi:10.1001/jama.299.4.425

14. Wittmann J, Jäck HM. Serum microRNAs as powerful cancer biomarkers. Biochim Biophys Acta 2010;1806:200-207. doi:10.1016/j. bbcan.2010.07.002

15. Gaedcke J, Grade M, Camps J, et al. The rectal cancer microRNAome-microRNA expression in rectal cancer and matched normal mucosa. Clin Cancer Res 2012;18:4919-4930. doi:10.1158/1078-0432.CCR-12-0016

16. Pucci S, Mazzarelli P. MicroRNA Dysregulation in Colon Cancer Microenvironment Interactions: The Importance of Small Things in Metastases. Cancer Microenviron 2011;4:155-162. doi:10.1007/s12307011-0062-y 
17. Raduly LZ, Braicu C, Jurj MA, et al. Dysregulations of circulating microRNAs are possible biomarkers for colorectal cancer. Eur J Cancer 2016; 61 Suppl 1:S114. doi:10.1016/S0959-8049(16)61404-1

18. Petrushev B, Boca S, Simon T, et al. Gold nanoparticles enhance the effect of tyrosine kinase inhibitors in acute myeloid leukemia therapy. Int J Nanomedicine 2016;11:641-660. doi:10.2147/IJN.S94064

19. Zaharie F, Muresan MS, Petrushev B, et al. Exosome-carried microRNA-375 inhibits cell progression and dissemination via Bcl-2 blocking in colon cancer. J Gastrointestin Liver Dis 2015;24:435-443. doi:10.15403/jgld.2014.1121.244.375

20. Jung G, Hernández-Illán E, Moreira L, Balaguer F, Goel A. Epigenetics of colorectal cancer: biomarker and therapeutic potential. Nat Rev Gastroenterol Hepatol 2020;17:111-130. doi:10.1038/s41575-019-0230-y

21. Wang ZH, Ren LL, Zheng P, et al. miR-194 as a predictor for adenoma recurrence in patients with advanced colorectal adenoma after polypectomy. Cancer Prev Res (Phila) 2014;7:607-616. doi:10.1158/1940-6207.CAPR-13-0426

22. Chang HY, Ye SP, Pan SL, et al. Overexpression of miR-194 Reverses HMGA2-driven Signatures in Colorectal Cancer. Theranostics 2017;7:3889-3900. doi:10.7150/thno.20041

23. Wang B, Shen Z, Gao Z, et al. MiR-194, commonly repressed in colorectal cancer, suppresses tumor growth by regulating the MAP4K4/ c-Jun/MDM2 signaling pathway. Cell Cycle 2015;14:1046-1058. doi:10 .1080/15384101.2015.1007767

24. Zhao HJ, Ren LL, Wang ZH, et al. MiR-194 deregulation contributes to colorectal carcinogenesis via targeting AKT2 pathway. Theranostics 2014;4:1193-208. doi:10.7150/thno.8712

25. Sundaram P, Hultine S, Smith LM, et al. p53-responsive miR-194 inhibits thrombospondin-1 and promotes angiogenesis in colon cancers. Cancer Res 2011;71:7490-7501. doi:10.1158/0008-5472.CAN-11-1124

26. Zhang Y, Dai J, Deng H, et al. miR-1228 promotes the proliferation and metastasis of hepatoma cells through a p53 forward feedback loop. Br J Cancer 2015;112:365-374. doi:10.1038/bjc.2014.593

27. Lin L, Liu D, Liang H, Xue L, Su C, Liu M. MiR-1228 promotes breast cancer cell growth and metastasis through targeting SCAI protein. Int J Clin Exp Pathol 2015;8:6646-6655.

28. Lee HO, Mustafa A, Hudes GR, Kruger WD. Hydroxychloroquine Destabilizes Phospho-S6 in Human Renal Carcinoma Cells. PloS One 2015;10:e0131464. doi:10.1371/journal.pone.0131464

29. Dieterlen MT, John K, Haase S, et al. Effect of confounding factors on a phospho-flow assay of ribosomal S6 protein for therapeutic drug monitoring of the mTOR-inhibitor everolimus in heart transplanted patients. Biomarkers 2017;22:86-92. doi:10.1080/13547 50X.2016.1210676

30. Choi JI, Park SH, Lee HJ, Lee DW, Lee HN. Inhibition of Phospho-S6 Kinase, a Protein Involved in the Compensatory Adaptive Response, Increases the Efficacy of Paclitaxel in Reducing the Viability of Matrix-Attached Ovarian Cancer Cells. PloS One 2016;11:e0155052. doi:10.1371/journal.pone.0155052

31. Grundy M, Jones T, Elmi L, et al. Early changes in rpS6 phosphorylation and $\mathrm{BH} 3$ profiling predict response to chemotherapy in AML cells. PloS One 2018;13:e0196805. doi:10.1371/journal.pone.0196805

32. Huynh FC, Nguyen D, Jones FE. Trastuzumab stimulation of ribosoma protein S6 kinase 1 [S6K1] predicts de novo trastuzumab resistance. Biochem Biophys Res Commun 2017;483:739-744. doi:10.1016/j. bbrc.2016.12.072

33. Fricke SL, Payne SN, Favreau PF, et al. MTORC1/2 Inhibition as a Therapeutic Strategy for PIK3CA Mutant Cancers. Mol Cancer Ther 2019;18:346-355. doi:10.1158/1535-7163.MCT-18-0510

34. Dromard M, Bompard G, Glondu-Lassis M, Puech C, Chalbos D, Freiss G. The Putative Tumor Suppressor Gene PTPN13/PTPL1 Induces Apoptosis through Insulin Receptor Substrate-1 Dephosphorylation. Cancer Res 2007;67:6806-6813. doi:10.1158/0008-5472.CAN-070513

35. Villa-Moruzzi E. PTPN12 controls PTEN and the AKT signalling to FAK and HER2 in migrating ovarian cancer cells. Mol Cell Biochem 2013;375:151-157. doi:10.1007/s11010-012-1537-y

36. Sengelaub CA, Navrazhina K, Ross JB, Halberg N, Tavazoie SF. PTPRN2 and PLC $\beta 1$ promote metastatic breast cancer cell migration through $\mathrm{PI}(4,5) \mathrm{P}_{2}$-dependent actin remodeling. EMBO J 2016;35:62-76. doi:10.15252/embj.201591973

37. Ozawa T, Kandimalla R, Gao F, et al. A MicroRNA Signature Associated With Metastasis of T1 Colorectal Cancers to Lymph Nodes. Gastroenterology 2018;154:844-848.e7. doi:10.1053/ j.gastro.2017.11.275

38. Kupcinskas J, Bruzaite I, Juzenas S, et al. Lack of association between miR-27a, miR-146a, miR-196a-2, miR-492 and miR-608 gene polymorphisms and colorectal cancer. Sci Rep 2014;4:5993. doi:10.1038/ srep05993

39. Steponaitiene R, Kupcinskas J, Langner C, et al. Epigenetic silencing of miR-137 is a frequent event in gastric carcinogenesis. Mol Carcinog 2016;55:376-386. doi:10.1002/mc.22287 